Casos Clínicos

Arch. Esp. Urol., 61, 6 (733-736), 2008

\title{
UTILIDAD DEL LITOTRIPTOR STONEBREAKERTM EN LA NEFROLITOTOMÍA PERCUTÁNEA
}

Almudena Coloma del Peso, Inmaculada Fernández González, Milagros Jiménez Gálvez, Pablo Garrido Abad, Gloria Bocardo Fajardo, Luis Miguel Herranz Fernández, Manuel Fernández Ariona, Lorenzo Herrero Torres e Ignacio Pereira Sanz.

Servicio de Urología. Hospital Universitario de La Princesa. Madrid. España.

Resumen.- OBJETIVO: La litiasis de la vía urinaria es una patología de gran prevalencia para cuyo tratamiento se han utilizado diversos dispositivos a lo largo de la historia. El StoneBreaker TM (LMA Urology, Gland, Switzerland) pertenece a una segunda generación de litotriptores intracorpóreos, neumático y portátil, que aporta algunas ventajas respecto a otros dispositivos, tanto por su eficacia en la fragmentación de las litiasis como por su fácil manejo, muy útiles durante la realización de la nefrolitotomía percutánea.

MÉTODOS/RESULTADOS: Presentamos el caso de un varón de 40 años con litiasis piélica de riñón izquierdo, al que se realiza nefrolitotomía percutánea bajo anestesia general, usando como litotriptor para la fragmentación de la misma el StoneBreaker ${ }^{T M}$ (LMA Urology, Gland, Switzerland).

Almudena Coloma del Peso

Servicio de Urología

Hospital Universitario de la Princesa

Diego de León, 62

28006 Madrid. (España).

almudenacoloma@hotmail.com

()

Trabajo recibido: 6 de noviembre 2007. 
CONCLUSIÓN: El StoneBreakerTM (LMA Urology, Gland, Switzerland) es un litotriptor para uso con endoscopios rígidos o semirrígidos, con mayor poder de fragmentación que sus antecesores, que consigue disminuir el número de disparos necesarios para la fragmentación de las litiasis, sin que esto repercuta en un aumento del daño tisular. Además también ha sido diseñado con una mayor comodidad de uso, gracias a que no precisa de conexiones y se acciona por medio de cartuchos desmontables de dióxido de carbono.

\section{Palabras clave: Stonebreaker. Litiasis. Litotriptor.}

Summary.- OBJECTIVE: Throughout the history, many devices have been used for breaking urinary tract stones. StoneBreaker ${ }^{T M}$ (LMA Urology, Gland, Switzerland) is a second generation of intracorporeal lithotripter, pneumatic and portable, which adds several new advantages, like effectiveness in stone fragmentation and easy handling, very useful during percutaneous lithotripsy.

METHODS/RESULTS: We report the case of a 40 year-old male pa-tient, with a left kidney pyelic lithiasis, who was treated by percutaneous lithotripsy, under general anaesthesia, with StoneBreaker ${ }^{T M}$ (LMA Urology, Gland, Switzerland) as lithotripter.

CONCLUSIONS: StoneBreaker'TM (LMA Urology, Gland, Switzerland) is a lithotripter usable with rigid and semirigid ureteroscopes, much more powerful than its predecessors. It is able to decrease the number of shocks necessary for stone fragmentation, without bigger tissue reaction. It also has a more comfortable design due to the absence of connections, and its power by replaceable carbon dioxi-de cartridges.
Keywords: Stonebreaker. Lithiasis. Lithotripter.

\section{INTRODUCCIÓN}

En la actualidad existen diferentes tipos de litotriptores intracorpóreos (electrohidraúlico, neumático, ultrasónico, electrocinético y láser). Algunas desventajas de estos sistemas, además del coste, son que requieren corriente eléctrica externa o que precisan del acceso a las tuberías comprimidas del aire y que no son portátiles. El StoneBreaker ${ }^{T M}$ (LMA Urology, Gland, Switzerland), es un nuevo dispositivo que es mucho más compacto y ergonómico que otros litotriptores neumáticos actuales y con mayor poder de fragmentación, generando presiones de contacto sobre la litiasis mayores a 29 bar, muy útil durante la realización de la Nefrolitotomía Percutánea (NLPC).

\section{CASO CLÍNICO}

Varón de 40 años de edad que consulta por presentar una litiasis piélica de riñón izquierdo con un diámetro de $4 \mathrm{~cm}$. Se le propone la realización de una NLPC. Bajo anestesia general y en posición de decúbito prono se accede al riñón por grupo calicial inferior, bajo control de escopia. Se dilata el trayecto de acceso percutáneo con dilatadores metálicos coaxiales de Alken. Se procede a la fragmentación de la litiasis con el litotriptor intracorpóreo StoneBreaker ${ }^{T M}$ (LMA Urology, Gland, Switzerland). El tiempo de fragmentación de la litiasis fue de 30 minutos utilizando tres cartuchos de dióxido de carbono comprimido (Figura 1A y 1B). No existieron
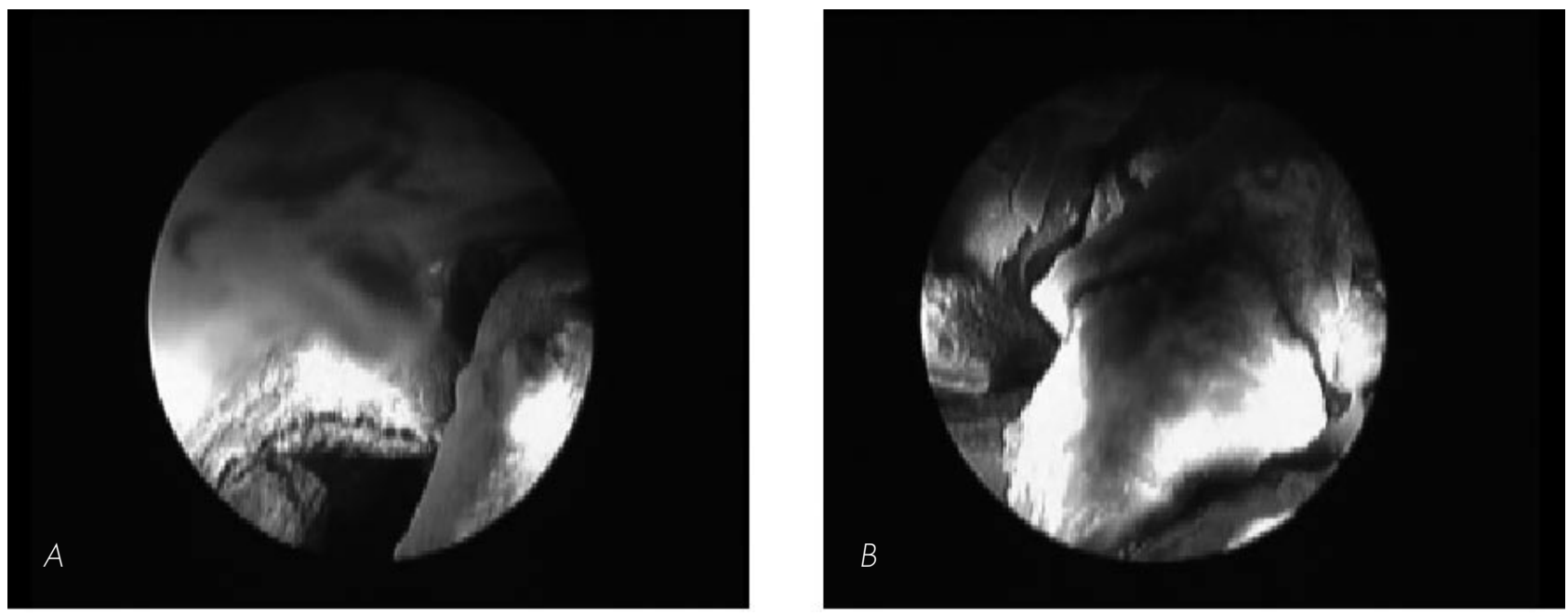

FIGURA IA. Imagen endoscópica donde se observa la sonda del litotriptor StoneBreaker ${ }^{\text {TM }}$ en el inicio de la NLPC. FIGURA IB. Final del proceso de fragmentación extrayendo los fragmentos litiásicos con pinzas de Nitinol de Cook. 
complicaciones y el paciente fue dado de alta a las 72 horas del procedimiento con un catéter doble J por la existencia de restos litiásicos menores de $3 \mathrm{~mm}$.

\section{DISCUSIÓN}

El StoneBreaker ${ }^{\mathrm{TM}}$ es un nuevo litotriptor intracorpóreo diseñado por los ingenieros que inventaron el Lithoclast $\AA^{\circledR}$. Esta "segunda generación" de litotriptor neumático es más compacta y ergonómica.

El StoneBreaker'TM (LMA Urology, Gland, Switzerland) es un litotriptor de contacto, neumático y portátil. Su peso es de aproximadamente 500 grs. y no precisa de conexiones eléctricas o neumáticas. El dispositivo es accionado por un cartucho desmontable con dióxido de carbono a alta presión ajustado para alcanzar un máximo funcionamiento operativo de 31 bar en el extremo de la sonda. Este extremo tiene un movimiento escaso, asegurando una fragmentación segura, sin riesgos y con mínima retropulsión (Figura 2).

Un cartucho de dióxido de carbono comprimido proporciona la energía necesaria para un procedimiento quirúrgico. Una vez que se ha activado el cartucho, el gas pasa a través de un regulador-reductor preajustado de presión para llenar un compartimiento intermedio. Se activa un martillo por resorte cuando se presiona el disparador; el gas comprimido se expande y de esta forma transmite energía al martillo; esto afecta a un proyectil dirigido, que transmite energía directamente a la punta de prueba. El gas expandido deja el compartimiento a través de un puerto y de una línea especialmente diseñados del extractor. Un resorte empuja el martillo de nuevo a su posición inicial de tal forma que el compartimiento intermedio se rellena con el gas y el dispositivo está preparado para un nuevo disparo. Con

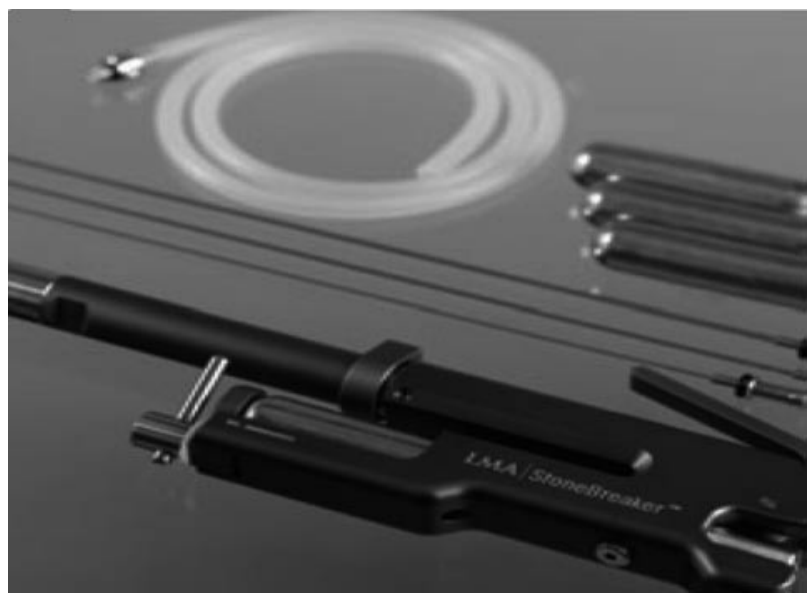

FIGURA 2. Fotografía del litotriptor StoneBreaker ${ }^{T M}$ con las sondas y los cartuchos de dióxido de carbono. un cartucho lleno se dispone de por lo menos de 80 disparos (Figura 3).

Se utiliza con endoscopios rígidos o semirrígidos que tengan un canal de trabajo recto, existiendo sondas de diferente calibre (1.0, 1,6 y $2.0 \mathrm{~mm})$.

Estudios de investigación animal utilizando el StoneBreaker $^{T M}$ para la fragmentación de cálculos artificiales alojados en el uréter inferior del cerdo, demuestran, con el estudio histológico de este segmento, la no existencia de una importante reacción tisular o perforación ureteral. Comparan también in vitro el Swiss Lithoclast ${ }^{\top M}$ con el StoneBreaker ${ }^{T M}$ concluyendo que se precisan menos disparos con el último litotriptor $(1,2)$.

Rané y cols. comunican su experiencia con la utilización del StoneBreaker ${ }^{T M}$ para la realización de la NLPC en 49 litiasis renales con un tamaño medio de $2.8 \mathrm{~cm}$. (rango 1,8-4,8). El número medio de disparos es de 34 (rango 2-76); no existieron complicaciones y el rango de stone free fue del $100 \%$ (3).

El StoneBreaker ${ }^{T M}$ es un litotriptor intracorpóreo neumático más robusto y efectivo que otros dispositivos existentes en la actualidad debido a que el número de disparos requeridos para obtener una adecuada fragmentación de la litiasis es menor; este parámetro es importante, teniendo en cuenta que una prolongada litotricia intracorpórea puede originar una peor visión durante el procedimiento endoscópico, con las consiguientes implicaciones en el resultado del mismo así como el riesgo potencial de originar un trauma tisular.

\section{CONCLUSIÓN}

El StoneBreaker ${ }^{T M}$ (LMA Urology, Gland, Switzerland) es un litotriptor para uso con endoscopios rígidos o semirrígidos, con mayor poder de fragmentación que sus antecesores, que consigue disminuir el número de disparos necesarios para la fragmentación de las litiasis, sin que esto repercuta en un aumento del daño tisular. Además también ha sido diseñado con una mayor comodidad

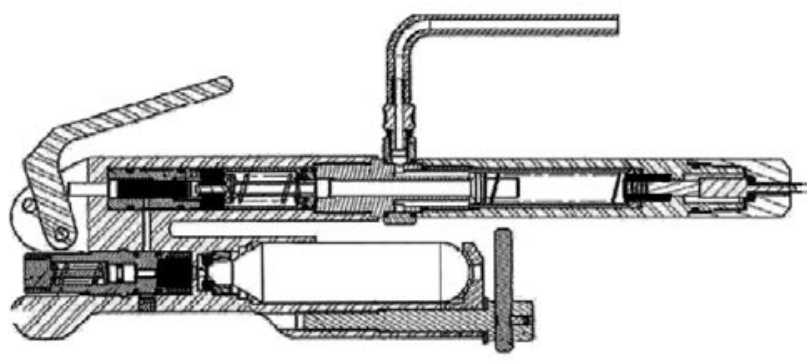

FIGURA 3. Esquema del mecanismo interno del litotriptor StoneBreaker ${ }^{T M}$ 
de uso, gracias a que no precisa de conexiones y se acciona por medio de cartuchos desmontables de dióxido de carbono.

\section{BIBLIOGRAFÍA Y LECTURAS RECOMENDADAS ( ${ }^{*}$ lectura de interés $y$ ** lectura fundamental)}

1. WADHWA, S.N.; HEMAL, A.K.; SHARMA, R.K.: "Intracorporeal lithotripsy with the Swiss lithoclast". Br. J. Urol., 74: 699, 1994.

2. RANÉ, A.; KYLE, C.; BHALANI, V. y cols.: "Further evaluation of the StoneBreaker ${ }^{\mathrm{TM}}$ : In vitro comparison with the Swiss Lithoclast ${ }^{\mathrm{TM}}$ ". Annual meeting of the British Association of Urogical Surgeons (BAUS), 2007.

3. RANÉ, A.; KOMMU, S.S.; KANDASWAMY, S.V. y cols.: "Initial clinical evaluation of a new pneumatic intracorporeal lithotripter”. BJU Int., 100: 629, 2007. 